\title{
Use of Structured Pattern Representations for Combining Classifiers
}

\author{
Raisa Socorro ${ }^{1}$ and Luisa Micó $^{2}$ \\ ${ }^{1}$ Instituto Superior Politécnico Jose Antonio Echevarría \\ La Habana, Cuba \\ ${ }^{2}$ Departamento de Lenguajes y Sistemas Informáticos \\ Universidad de Alicante \\ P.O. box 99, E-03080 Alicante, Spain
}

\begin{abstract}
In Pattern Recognition, there are problems where distinct representations can be obtained for the same pattern, and depending on the type of classifiers (statistical or structural) one type of representation is preferred versus the others. In the last years, different approaches to combining classifiers have been proposed to improve the performance of individual classifiers. However, few works investigated the use of structured pattern representations. In this paper combination of classifiers has been applied using tree pattern representation in combination with strings and vectors for a handwritten character classification task. In order to save computational cost, some proposals based on the use of both embedding structured data and refine and filter framework are provided.
\end{abstract}

Keywords: combining classifiers, tree representation, string representation, edit distance, $\mathrm{k}-\mathrm{NN}$ rule.

\section{Introduction}

Multiple classifier systems have received much attention in the recent years. Its success is in the use of different decisions to obtain a more accurate classification than single classifiers. Classifier ensemble has mainly two advantages: on the one hand, the recognition performance is higher in opposite of single classifiers. On the other hand, the behavior of a multiple classifier system is more robust due to the fact that a final decision is taken on the basis of several decisions (critical individual decision can be avoided) [1]. As stated in a recent work by Duin [2], a consistent set of different classifiers should be used, but also they should be comparable. In the same work different sets of consistent combined classifiers are presented (using different initializations, parameters choice, classification schemes, etc) obtaining the best results when different feature sets have been used.

While regular vector-space represented data are widely used for combining classifiers, structured data have been rarely used. The main reason is the wide range of different classification methods that can be applied when patterns are represented as vectors. However, the number of classifiers to use is drastically 
reduced for structural pattern representations. For example, in the case of structured data (strings, trees, graphs ...), if a dissimilarity measure is defined, a $k$ nearest neighbor $(k$-NN) classifier can be applied. Also, recently, the use of kernel functions based on edit distances [3] or the use of embedding for structured data in real vector spaces [4] have enabled the use of alternative statistical methods for classification.

Structural representation of data have been successfully used in many recognition tasks. In fact, it is the more natural representation of data in many applications, as for example in computational biology. The main advantages of using a structural representation is that the number of features is not fixed and the relationship between individual feature components appears explicitly in the representation (in contrast with the feature vector representation).

In this work we analyse how the combination of more than a structural representation can improve the classification error rate. As well as strings and vectors have been used previously in classifier ensembles, trees have not been used ever. The experiments performed show that the performance of the classification improves when trees are used in combination with vectors and strings. Moreover, experiments performed using embedding strings and vectors and a "filter and refine" classifier show that a significant speedup can be achieved at the expense of a decrease in the rate of success. In all the experiments, the classifier we adopted is the $k$-NN. As different representations are used, different dissimilarity measures are needed, associated to the type of data. For that reason, in this work the Euclidean distance (ED), the string-edit-distance (SED) and the tree-edit-distance (TED) have been used.

In the following sections, feature extraction methods for data and ensemble schemes used in this work are described. Then, the results for the ensembles are presented for binary images of handwritten characters from the NIST 3 Database National Institute of Standards and Technology [5]. Finally, the conclusions drawn from the results are discussed, pointing the research to further work lines.

\section{Feature Extraction of the Data Set and Distances}

Three different feature extraction methods have been used in this work, two of them are structural representations (trees and strings) and the third is a representation in a real vector space. Given a pattern image, the key idea is that each representation gives some type of information about the pattern that it is not given by the others. For example, strings represent the contour of the images, trees represent the skeleton, and each dimension in a $n$-dimensional vector represents the ratio of black pixels over the total in a region of the image.

\subsection{Tree Data Representation}

The first step before obtaining a tree data representation of patterns is the use of a thinning algorithm to eliminate redundant information in the image [6]. 
The image is scanned from left to right starting from the top looking for the first black pixel. This first pixel is marked with label "0". Every tree node has so many descendents as unmarked neighbors has the selected and marked pixel; each of the new branches is extended following their neighborhood until: a) the branch has a maximum size (parameter $R$ of the algorithm 1 ), b) is a terminal pixel or c) is an intersection pixel. After that, a new node is assigned to every end of branch. The labels of the nodes (except for the root) have values between " 1 " and " 8 " depending on the direction of the branch (see figure 1 on the left). More detailed information about this algorithm can be found in 7 .
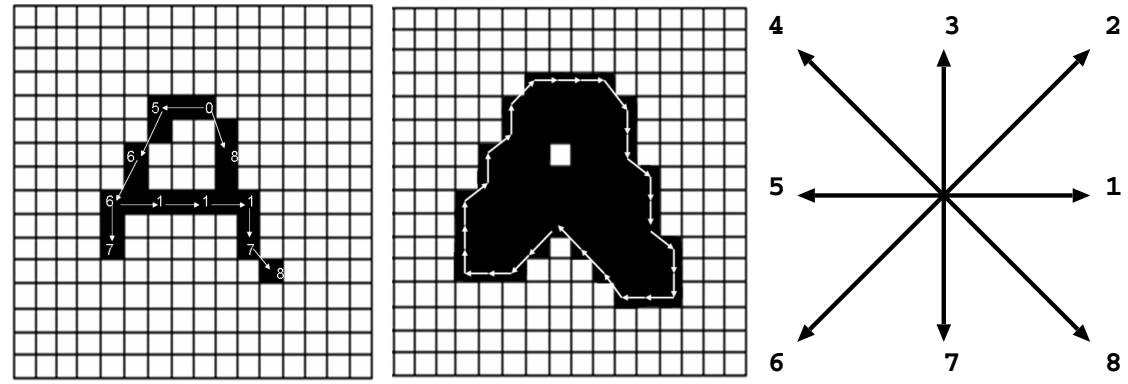

Fig. 1. On the left, example of a tree and a contour string. On the right, symbols used to encode strings and trees

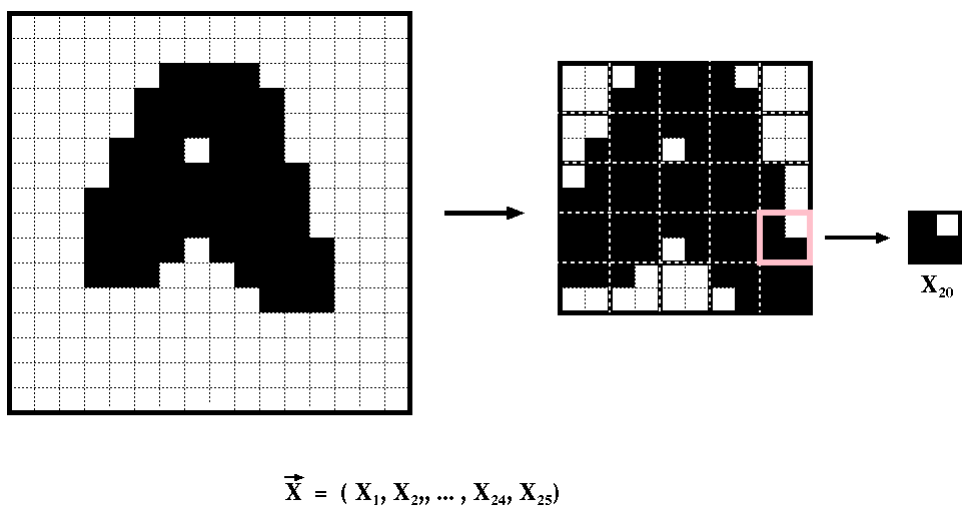

Fig. 2. Example of a vectorial data representation

A general TED is defined by Shasha and Zhang in 8 . Given trees $T_{1}$ and $T_{2}$, a dynamic programming algorithm with space complexity $O\left(\left|T_{1}\right| \times\left|T_{2}\right|\right)$ and $O\left(\left|T_{1}\right| \times\left|T_{2}\right| \times \min \left(\operatorname{depth}\left(T_{1}\right) ;\right.\right.$ leaves $\left.\left(T_{1}\right)\right) \times \min \left(\operatorname{depth}\left(T_{2}\right) ;\right.$ leaves $\left.\left(T_{2}\right)\right)$ in time

${ }^{1}$ In this work the parameter $R=2$, that means that the maximum length of each branch of the tree is 2 pixels. 
complexity has been used in this work. For the edit basic operations (insertion, deletion and substitution of nodes) in the tree, the following weights were used:

$-w_{i}=w_{d}=1$ (insertion and deletion weights)

$-w_{i j}=\min (|i-j| ; 8-|i-j|)$ (substitution weights)

The distance $d_{t}\left(T_{1}, T_{2}\right)$, is finally normalized (to avoid the size invariance problem) with the maximum size of the two trees 2 :

$$
d_{N}\left(T_{1}, T_{2}\right)=\frac{d_{t}\left(T_{1}, T_{2}\right)}{\max \left(\left|T_{1}\right|,\left|T_{2}\right|\right)}
$$

\subsection{String Data Representation}

Following the same strategy than in the previous method, the image is scanned from left to right starting from the top, searching the first black pixel 3 . In this case, the external contour of the characters (the string) is obtained going to the right (in the clockwise sense) until the first pixel is reached again (see figure 1 in the middle). The symbols (directions) in the strings are the same than in the case of trees. The edit distance between two strings $S_{1}$ and $S_{2}, d_{s}\left(S_{1}, S_{2}\right)$, is defined as the minimum-cost set of transformation that must be done to turn a string into the other. The edit basic operations are insertions, deletions and substitution of individual symbols in the string. The cost values for the operations are equal that those used in TED. This distance can be computed in time $O(|x| \times|y|)[9$. As in trees, the distance is finally normalized with the maximum of the length of the strings to avoid the size invariance problem:

$$
d_{N}\left(S_{1}, S_{2}\right)=\frac{d_{s}\left(S_{1}, S_{2}\right)}{\max \left(\left|S_{1}\right|,\left|S_{2}\right|\right)}
$$

\subsection{Vectorial Data Representation}

Firstly, to obtain a size invariance representation of characters, the smallest squared matrix that includes the character in the original image is selected (see Figure 2). The scaled image is divided in $n$ fixed squared windows, where $n$ will be the dimension of a vector, and each dimension of this vector represents the ratio of black pixels in relation to the total number of pixels in each window. After some preliminary experiments, the best results were obtained using 25dimensional vectors.

\section{Classifier Ensembles}

Designing a suitable method of decision combinations is a key point for the ensemble's performance. In this paper, two different combination schemes have

${ }^{2}$ Different normalizations have been applied, but in this work only results with the best behavior are shown.

${ }^{3}$ In order to reduce the length of the strings, all the images were scaled from $128 \times 128$ to $64 \times 64$. 
been employed, one of them is based on a confidence voting scheme (sum rule) [10] and the second is a scheme based on the filter-and-refine framework [11].

\subsection{Confidence Voting Approach}

In confidence voting methods, voters express the degree of their preference for a candidate. This is done by assigning a value (called the confidence value) to the candidate. The higher the confidence value, the more the candidate is preferred by the voter [1].

Although different confidence methods have been applied (some of them can be found in [12 [13]) in this work the best results have been obtained with the following confidence:

$$
C_{i}(x)=\frac{\sum_{i} \frac{1}{d_{i}(x)^{2}}}{\sum_{j=1}^{k} \frac{1}{d_{j}(x)^{2}}} \quad i: 1 \ldots C
$$

where the $k$-nearest distances to the sample $x$ have been used. In particular $\sum_{i} \frac{1}{d_{i}(x)^{2}}$ is the sum of the inverse squared distances of the nearest neighbors belonging to the class i among the $k$ nearest neighbors.

Kittler et al. performed in [10] an experimental comparison of different classifier combination schemes. They show that the combination rule called sum rule outperforms others combination schemes when different pattern representation of data are used. For this reason in this work we have used this combination (even so, others combination schemes were also tested with worst results).

Sum rule: each voter has to give a confidence value for each candidate. Next, all confidence values are added for each candidate and the candidate (the class) with the highest value of the sum is selected.

\subsection{Filter and Refine Schemes}

In domains where the distance measure is computationally expensive, different approaches to accelerate the search have been proposed. For example, approximate cascade classifiers can been used [14]. In this scheme normally inaccurate but cheap classifiers are considered, followed by the more accurate and expensive, ie, the idea is to use at different levels different classifiers (starting by the less expensive). The number of levels depends on the problem and different possibilities can be build for the same problem.

On the other hand, embedding methods have been recently proposed for speeding up the search [11. These methods embed the strings or trees (or any structural object for which a distance has been defined) in a vector space, so the distances of the embedded objects approximates the actual distances. Thus, the search can be performed on the embedded objects using, for example, the ED.

Embedding methods can be used in a "filter and refine" scheme to accelerate the search [14]. This method consists on: 
- firstly, the sample in the embedding space is used as a "filter", where a small set of candidates are selected (the most promising);

- secondly, a new classification in the original space is performed using only the selected set to "refine" the result 4 .

In this work, two different two-level "filter and refine" schemes have been defined based on the use of these ideas.

\section{Experiments}

To perform the experiments, binary images of handwritten segmented uppercase characters from the NIST 3 Database National Institute of Standards and Technology were used (see some examples in figure 33):

a) number of classes in this task: 26

b) types of representation: 25-dimensional vectors, strings and trees

c) distances: ED, SED and TED

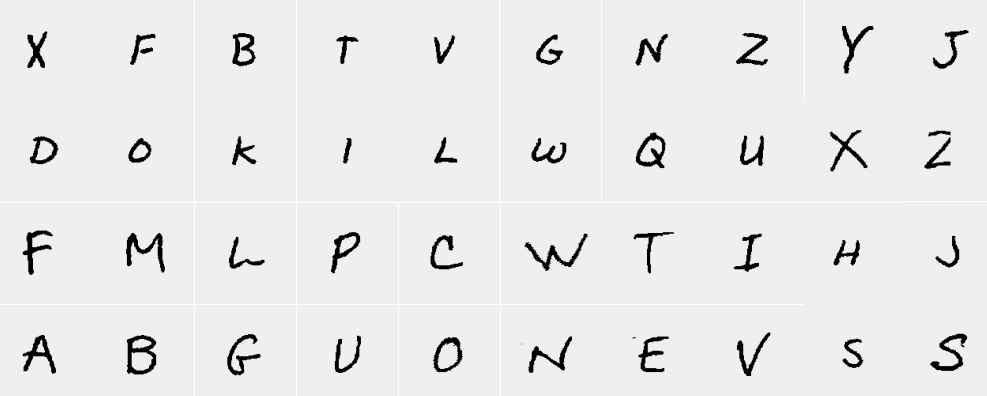

Fig. 3. Some examples of upper handwritten characters from the NIST Database 3

Each experiment were repeated 10 times using training and test sets with 1080 samples on average.

To perform a combination based on the confidence voting scheme, the results of the $k$-NN rule of individual classifiers with different representations of data has been used as input. In particular, the following combinations have been performed:
C1: strings and vectors
$\mathrm{C} 2$ : strings and trees
C3: trees and vectors
C4: strings, trees and vectors

\footnotetext{
${ }^{4}$ In practice, this two steps can be interleaved.
} 
Table 1. Average classification error using the $k$-NN rule (standard deviation are in brackets) using strings, trees and vectors and four combinations

\begin{tabular}{|c|c|c|c||c|c|c|c|}
\cline { 2 - 8 } \multicolumn{1}{c|}{} & \multicolumn{3}{c||}{ individual classifiers } & \multicolumn{4}{c|}{ classifier ensembles } \\
\hline $\mathbf{k}$ & strings & trees & vector & C1 & C2 & C3 & C4 \\
\hline $\mathbf{1}$ & $\mathbf{1 2 . 9 ( 1 . 1 )}$ & $26.4(1.6)$ & $27.4(1.4)$ & $19.6(1.0)$ & $19.4(1.4)$ & $26.5(1.1)$ & $13.3(1.1)$ \\
\hline $\mathbf{3}$ & $13.8(1.3)$ & $27.4(1.8)$ & $28.9(1.2)$ & $11.0(1.0)$ & $11.5(1.3)$ & $16.7(1.3)$ & $\mathbf{0 8 . 9 ( 1 . 1 )}$ \\
\hline $\mathbf{5}$ & $15.9(1.6)$ & $30.4(1.8)$ & $32.4(1.0)$ & $10.5(0.7)$ & $10.9(1.4)$ & $15.8(1.5)$ & $09.1(1.0)$ \\
\hline $\mathbf{7}$ & $18.0(1.6)$ & $33.2(2.0)$ & $35.6(1.0)$ & $10.5(0.6)$ & $11.1(1.5)$ & $16.2(1.7)$ & $09.3(1.1)$ \\
\hline
\end{tabular}

Table 1 shows the classification error rate (in \%) when the $k$-NN classifier is used for the three types of representation. The first three columns show the result of the individual classifiers for each representation. In this case, the best results are obtained when the $k$-NN classifier is used with $k=1$, and strings outperforms significantly trees and vectors. The four columns on the right of table show the classification error rate for the ensembles. In this case, it can be observed that the classification error rate is influenced by the number of nearest neighbors $k$, and depends on the combination. Some interesting results are obtained: firstly, depending on the combination, the best results were obtained for $k$ values greater than 1 ; secondly, any combination of two representation were strings are represented (C1 and $\mathrm{C} 2$ ) improves the results of any individual classifier; thirdly, the best results are obtained using combination $\mathrm{C} 4$ with $k=3$ (using the three representations).

As the use of edit distance with strings or trees is very time consuming, other alternatives have been analyzed trying to reduce it. In particular, experiments with two schemes based on refine and filter classifiers and embedding methods were done.

Embedding strings and trees. Strings and trees were embedded as vectors using the edit distance to a selected number of prototypes. That is, an object (string or tree) can be transformed into a vector by calculating the edit distance to all the selected objects, where each distance represents one vector component. Formally, given a set of objects $P$ and a subset $B=\left\{b_{1}, \ldots, b_{n}\right\} \subseteq P$, the transformation $t_{n}^{B}: P \rightarrow \mathbb{R}^{n}$ is defined as a function where $t_{n}^{B}(x)=\left(d\left(x, b_{1}\right), \ldots, d\left(x, b_{n}\right)\right)$, and where $d\left(x, b_{i}\right)$ is the edit distance between the objects $x$ and $b_{i}$.

Spillman et al. proposed in [4] some prototype selection methods to use 5 . The method used in this work was the following: the first one, $b_{1}$, is randomly selected among $P$ and then, for $i=2,3 \ldots n$

$$
b_{i}=\operatorname{argmax}_{p \in\left(P-B_{i}\right)} \min _{k=1}^{i-1} d\left(p, b_{k}\right),
$$

where $B_{i}=\left\{b_{1}, \ldots, b_{i-1}\right\}$.

For every $p \in P$, the edit distance from these $n$ selected objects are computed, and these $n$ distances are used as the $n$ coordinates of each object $p$ in a new

${ }^{5}$ These methods are similar than the used in some fast nearest search algorithms based on prototype selection [7]. 
Table 2. Average classification error (standard deviation are in brackets) using different number of reference points in the embedding for $k=5$

\begin{tabular}{|c|c|c|c|c|}
\cline { 2 - 5 } \multicolumn{1}{c|}{} & \multicolumn{4}{c|}{ dimension } \\
\hline type & $\mathbf{5 0}$ & $\mathbf{1 0 0}$ & $\mathbf{1 5 0}$ & $\mathbf{2 0 0}$ \\
\hline strings & $21.2(1.2)$ & $19.8(1.1)$ & $18.9(1.0)$ & $18.7(0.9)$ \\
trees & $30.0(1.3)$ & $30.1(1.0)$ & $30.3(1.0)$ & $30.0(1.0)$ \\
\hline
\end{tabular}

Table 3. Average classification error (standard deviations are in brackets) using the $k$-NN rule with individual and combined embedded strings, trees and vectors

\begin{tabular}{|c|c|c|c||c|}
\cline { 2 - 5 } \multicolumn{1}{c||}{} & \multicolumn{3}{c||}{ individual classifiers } & combining classifiers \\
\hline $\mathbf{k}$ & Emb_string & Emb_trees & vector & C5 \\
\hline $\mathbf{1}$ & $22.2(2.2)$ & $31.4(1.4)$ & $27.4(1.4)$ & $18.0(1.2)$ \\
$\mathbf{3}$ & $21.4(1.8)$ & $30.5(1.1)$ & $28.9(1.2)$ & $12.6(1.3)$ \\
$\mathbf{5}$ & $21.2(1.2)$ & $30.0(1.3)$ & $32.4(1.0)$ & $\mathbf{1 2 . 1 ( 1 . 0 )}$ \\
$\mathbf{7}$ & $21.5(1.3)$ & $30.3(1.2)$ & $35.6(1.0)$ & $12.4(1.3)$ \\
\hline
\end{tabular}

transformed $n$-dimensional space. The experiment presented in Table 2 were performed to select the dimensionality in the transformed vector space.

The combination with the best behavior in Table 1 (C4: combining strings, trees and vectors) were repeated using the embedding of trees and strings (for $n=50$ ). The results can be shown in Table 3 . Except for $k=1$, for the other values of $k$ we found a better result using the new combination (called C5) than the better individual classifier with the difference that now the classification is faster that in the individual classifiers.

Refine and filter classifiers. Two schemes were applied:

EmbS-OrS: Firstly, a $k$-NN rule is used with the embedded strings in a 50dimensional vector space using the ED. Secondly, the 100 nearest samples to the test were used as training set for the second classifier. For this second classifier, the original strings using the SED were used.

Vec-OrS: Firstly, a $k$-NN rule is used with the 25-dimensional vector representation obtained in section 2.3 using the ED. Secondly, the 200 nearest samples to the test were used as training set for the second classifier. For this second classifier, the original strings using the SED were used.

Table 4 shown results with both schemes. It can be observed that the best results are obtained when the embedding method is used in the first classifier.

In this work, the main goal for using embedding and refine and filter classifiers were the reduction of the time complexity. Table 5 shows this reduction (see the last three columns) were for the combination C5 (using the embedding of strings, trees and the vector representation), slightly reduces the error rate obtained by the contour string, but also reducing up to $8.0 \%$ the classification time. However, if our main goal is to reduce the error rate, the combination $\mathrm{C} 4$ can be used. In our experiments, we have reduced the error from $12.9 \%$ to $8.9 \%$. 
Table 4. Average classification error (standard deviations are in brackets) for a filter and refine framework

\begin{tabular}{|c|c|c|c|}
\hline Second class. & EmbS-OrS & Vec-OrS & Contour string \\
\hline 1 & $13.2(1.2)$ & $13.6(1.2)$ & $12.9(1.1)$ \\
3 & $12.3(0.9)$ & $14.5(1.4)$ & $13.8(1.3)$ \\
5 & $12.4(1.1)$ & $16.5(1.7)$ & $15.9(1.6)$ \\
7 & $12.9(1.2)$ & $18.5(1.7)$ & $18.0(1.6)$ \\
\hline
\end{tabular}

Table 5. Classification error and time for all combinations. Contour string error rate and time is used as baseline.

\begin{tabular}{|c|c|c|c|c|c|}
\cline { 2 - 6 } \multicolumn{1}{c|}{} & Contourn string & C4 & C5 & EmbS-OrS & Vec-OrS \\
\hline error (\%) & 12.9 & $\mathbf{8 . 9}$ & 12.1 & 12.3 & 13.6 \\
\hline time (\%) & 100 & 158 & $\mathbf{8}$ & 13.9 & 18.37 \\
\hline
\end{tabular}

\section{Conclusions}

In this work classifier ensembles using structural information of data have been applied in a handwritten character recognition task. Different ensembles have been evaluated, some of them to improve the classification rate and others to reduce the time complexity. The proposed schemes improve the classification rate or the time consuming in relation to the individual classifiers. To reduce the time complexity a scheme based on the use of a refine and filter framework an embedding methods have been used, reducing this time until a $8 \%$ maintaining similar error rate that the best individual classifier. The combination of trees, strings and vectors reduce the error rate from $12.9 \%$ to $8.9 \%$ when a training set of 1080 samples on average is used (but at expense of consuming more time). In future work, new schemes using alternative embedding methods and fast approximated nearest neighbors algorithms will be analyzed to reduce both the error rate and the time complexity.

\section{Acknowledgments}

This work was supported by the Spanish project CICyT DPI2006-15542-C04-01 and the IST Programme of the European Community, under the Pascal Network of Excellence, IST-2002-506778.

\section{References}

1. Kuncheva, L.: Combining Pattern Classifiers: methods and algorithms. Wiley, Chichester (2004)

2. Duin, R.: The combining classifier: to train or not to train? In: Proceedings of the International Conference on Pattern Recognition ICPR 2002, Quebec Canada, vol. II, pp. 765-770 (2002) 
3. Neuhaus, M., Bunke, H.: Edit distance-based kernel functions for structural pattern classification. Pattern Recognition 39(10), 1852-1863 (2006)

4. Spillmann, B., Neuhaus, M., Bunke, H., Pekalska, E., Duin, R.: Transforming strings to vector spaces using prototype selection. In: Yeung, D.-Y., Kwok, J.T., Fred, A., Roli, F., de Ridder, D. (eds.) SSPR 2006 and SPR 2006. LNCS, vol. 4109, pp. 287-296. Springer, Heidelberg (2006)

5. Grother, P.: Handprinted forms and character database. In: NIST Special Database 19 (2001)

6. Carrasco, R., Forcada, M.: A note on the Nagendraprasad-Wnag-Gupta thinning algorithm. Patter Recognition Letters 16, 539-541 (2003)

7. Rico, J., Micó, L.: Comparison of aesa and laesa search algorithms using string and tree edit distances. Pattern Recognition Letters 24(9), 1427-1436 (2003)

8. Zhang, K., Shasha, D.: Simple fast algorithms for the editing distance between trees and related problems. SIAM J. Comput. 18, 1245-1262 (1989)

9. Wagner, R., Fischer, M.: The string-to-string correction problem. Journal of the Association for Computing Machinery 211, 168-173 (1974)

10. Kittler, J., Hatef, M., Duin, R., Matas, J.: On combining classifiers. IEEE Trans. on Pattern Analysis and Machine Intelligence 20, 226-239 (1998)

11. Hjaltason, G., Samet, H.: Properties of embedding methods for similarity searching in metric spaces. IEEE Trans. on Pattern Analysis and Machine Intelligence 25, 530-549 (2003)

12. Duin, R.P.W., Tax, D.M.J.: Classifier conditional posterior probabilities. In: Amin, A., Pudil, P., Dori, D. (eds.) SPR 1998 and SSPR 1998. LNCS, vol. 1451, pp. 611619. Springer, Heidelberg (1998)

13. Arlandis, J., Perez-Cortes, J.C., Cano, J.: Rejection strategies and confidence measures for a k-nn classifier in an ocr task. In: 16th International Conference on Pattern Recognition ICPR 2002, pp. 576-579. IEEE, Los Alamitos (2002)

14. Athitsos, V., Alon, J., Sclaroff, S.: Efficient nearest neighbor classification using a cascade of approximate similarity measures. In: Proceedings of the 2005 IEEE Computer Society Conference on Computer Vision and Pattern Recognition (CVPR 2005), vol. 1, pp. 486-493 (2005) 